interested in non-pharmacological therapies to symptoms (O'Regan \& Filshie, 2010). Acupuncture offers relief from both physical and psychological symptoms often experienced in this patient group. Acupuncture was introduced to the hospice as a non-pharmacological option for both in-patients and outpatients in 2018.

Aims To monitor the effects of acupuncture on a range of palliative symptoms using visual analogue scales (VAS), patient satisfaction surveys and verbal feedback.

Methods Patients who may benefit from acupuncture for a variety of symptoms were identified by the multidisciplinary team. Acupuncture points were selected by an AACP trained physiotherapist following full assessment, health screen and written informed consent. Symptoms were monitored using VAS and feedback forms prior to each session of acupuncture with each patient.

Results $87 \%$ of patients who were identified by the MDT for acupuncture consented to trying this therapy in conjunction with other medical treatment. Positive results in reducing symptoms using VAS were shown in patients presenting with pain (mean VAS pre-treatment 8.7, mean VAS post-treatment 6.7) nausea (mean pre-treatment VAS 7.6, mean VAS, posttreatment 2.3) breathlessness (mean pre-treatment VAS 7.3, mean VAS, post-treatment 6.3) fatigue (mean pre-treatment VAS 8.6, mean VAS, post-treatment 6.3) and anxiety (mean pre-treatment VAS 7.6, mean VAS, post-treatment 3.6). Subjectively $70 \%$ of patients reported that acupuncture was a positive experience regardless of whether the primary symptom had improved. Patients reported feeling calmer and more relaxed during and after sessions.

Conclusion Acupuncture has proved to be a popular, safe and enjoyable adjunct to therapy intervention for patients with no adverse reactions or side effects of treatment. Early results have shown quantitative improvements in VAS as well as qualitative positive results for the use of acupuncture in patients' emotional and psychological wellbeing.

\section{P-167 REFLEXOLOGY IN PALLIATIVE CARE: REMOVE THE PLACEBO EFFECT AND WHAT'S LEFT?}

\footnotetext{
${ }^{1,2}$ Simon Noble, ${ }^{2}$ Alana Douglas-Jones, ${ }^{2}$ Alice Dawson. ${ }^{1}$ Marie Curie Palliative Care
} Research Centre, Cardiff, UK, ${ }^{2}$ Hospice of the Valleys, Ebbw Vale, UK

\subsection{6/bmjspcare-2019-HUKNC.189}

Background The placebo effect may result in a $40 \%$ clinical improvement following administration of a non-active treatment. Complementary therapies such as reflexology are commonplace in palliative care services yet their evidence base is supported by poorly constructed research and subjective clinical outcomes. Cynics have attributed patient-reported responses to the placebo effect.

Method Retrospective data analysis of hospice patients' pre and post symptom-targeted reflexology using the palliative care outcome scale (POS-S) evaluation tool. Mean symptom difference (MSD) and percentage response (PR) were calculated for each symptom. Placebo adjusted response (PAR) was calculated (PR-40\%) with PAR of $20 \%$ or above being considered significant.

Results 100 hospice patients received a total of 360 treatment sessions for eight symptoms. (See Abstract 167, Table 1).

Conclusion Adjusting patient-reported response for placebo effect suggests reflexology may have a positive effect on

\begin{tabular}{lccc}
\multicolumn{4}{l}{ Abstract P-167 Table 1} \\
Symptom & MSD & PR & PAR \\
\hline Pain & 0.74 & $24 \%$ & $<0$ \\
Constipation & 1.5 & $80 \%$ & 40 \\
Insomnia & 0.8 & $40 \%$ & 0 \\
Distress & 0.8 & $47 \%$ & 7 \\
Neuropathy & 0.8 & $47 \%$ & 7 \\
Fatigue & 1.6 & $80 \%$ & 40 \\
Dyspnoea & 0 & $0 \%$ & 0 \\
Poor appetite & 0.25 & $17 \%$ & $<0$ \\
\hline
\end{tabular}

constipation and fatigue. Constipation has long been reported to respond to reflexology and can be objectively evaluated with validated outcome tools. A feasibility study to conduct a randomised controlled trial to compare reflexology with placebo for constipation is in development.

\section{P-168 THE RELAXATION REVOLUTION - A HOSPICE BASED RELAXATION GROUP TO SUPPORT PATIENTS WITH SOME 'R AND R'}

Angeliki Panteli. St Wilfrid's Hospice, Eastbourne, UK

\subsection{6/bmispcare-2019-HUKNC.190}

Background There has been increased use of non-pharmacological treatments to support patients with life-limiting illness. Relaxation is now seen as a core component of interventions in hospice and palliative care (Miller \& Hopkinson, 2008). The benefits have been well documented. A weekly hospice complementary therapist-led relaxation group was introduced and its impact evaluated.

Aim To evaluate the relaxation group and assess whether relaxation techniques help patients' symptoms and improve their sense of wellbeing.

Method Patients attended a six-week relaxation programme where they learnt techniques including breath-work, mindfulness meditations, guided visualisations and progressive muscle relaxation techniques. Referrals were received from within the hospice.

Using an adapted Visual Analogue Scale, each week patients were asked to identify their main symptom with a score from 0 (no symptom) to 10 (the worst it could be) pre- and postintervention. Patients were also asked to rate their wellbeing on a scale from $0 \%$ (no sense of wellbeing) to $100 \%$ (the best they could feel) pre- and post- session. Results were further analysed to see which symptoms reported were related to an improvement in wellbeing.

Results Out of 121 patient contacts over 27 weeks, 113 (93\%) reported an improvement in their symptoms. Overall mean before was 6.3 and post 3.5 (improvement of 2.8). Stress improved by a mean of 4.1 ; pain by 3.3 ; anxiety by 2.9 and Shortness of Breath (SOB) by 2.8.

A total of $98(81 \%)$ reported an improvement in sense of wellbeing. Results were further analysed to see which symptoms reported had a correlated improvement in wellbeing. One example: patients citing $\mathrm{SOB}$ as main symptom felt their wellbeing improved by $20 \%$.

Conclusion The relaxation group showed benefit to hospice patients and empowered them to use a holistic tool to manage 
their health and wellbeing. The study can be used as a patient-centred approach for future planning and service provision.

\section{P-169 EFFECT OF A HOLISTIC APPROACH TO THE USE OF AQUATIC THERAPY FOR PALLIATIVE ADULT PATIENTS}

Charlotte Nicklin. Keech Hospice Care, Luton, UK

10.1136/bmjspcare-2019-HUKNC.191

\section{Background}

- Aquatic therapy is not standardly provided for palliative patients;

- A hydrotherapy pool provides a warm supportive environment for painful deconditioned bodies during or after medical treatment;

- Provides an opportunity to move, strengthen, relax and participate with significant effect on emotional well-being;

- Extends patient opportunities to therapies outside medical treatment, putting patients back in control.

Aims To evaluate the provision of aquatic therapy (therapistled hydrotherapy) for palliative patients by comparing objective measures and goals pre- and post- treatment

- Provide therapist-led aquatic therapy;

- Nurture and empower patients to become independent pool users.

Method Assess and work with palliative adult patients delivering aquatic therapy in 1:1 or group settings.

- Identify patient goals, physical baseline of ability including a range of standardised physical objective measures, patient perception of issues and well-being;

- 1:1 water-based assessment explores;

$\circ$ patient ability to exercise in water

○ water confidence

- trial personalised exercises and develop own written programme

- $<6$ further sessions of aquatic therapy 1:1, group or combination;

- Patient feedback questionnaire completed.

Results Data collected from all patients who completed a course of aquatic therapy, average duration two months.

- $100 \%$ improved physical function;

- $83 \%$ improved emotional well-being;

- $92 \%$ continued as independent pool users.

Conclusion Aquatic therapy can have a very positive, significant effect for patients affecting their physical function, willingness and motivation for exercise through their own perception of improvement, and emotional well-being. Relatively simple, short periods of interaction by a therapist can have meaningful long-term benefits for palliative adult patients for both physical outcomes and emotional well-being.

Innovation

- Currently no standardised use of aquatic therapy study demonstrates very positive outcomes for palliative patients including their perception, improved physical changes and function;

- Emphasis to empower patients with independent pool sessions in addition to attending a therapist-led session;
- Patients feel they are doing something to help themselves, which they find rewarding and motivating.

\section{P-170 MANAGING A CHANGE PROJECT - HOSPICE HYDROTHERAPY POOL}

Clare Burden. Keech Hospice Care, Luton, UK

10.1136/bmjspcare-2019-HUKNC.192

Background Hospice patient use of the hydrotherapy pool for aquatic therapy and memory work was minimal due to lack of information, unclear referral processes and no patient pathways for hydrotherapy.

Aims Increase hospice patient use of the pool by introducing clear referral pathways and having a structured Hydrotherapy pool team (Administrator and Lifeguards). For processes to be introduced that enabled individual members of the public with an appropriate need for hydrotherapy to access the pool and for external organisations to only use required spaces.

Methods Processes and access to the hydrotherapy pool by patients and their families, individual members of the public and external organisations were reviewed. Pool user expectations were managed, and changes communicated throughout. The structure and skill mix of the hydrotherapy pool team was reviewed and restructured. Care teams were involved in developing the pathways and referral process. Final versions of pathways were cascaded to teams.

Results Patient referrals for using the pool have increased and benefits are being recorded by the Aquatic Physiotherapist. It is expected that patient numbers will increase further. There is an increase in individual members of the public using the pool appropriately, through referrals from physiotherapists. External organisations are booking required number of spaces and confirming prior to use, thus allowing more patient and family spaces in the pool.

Conclusions Hospice patients and their families are a priority for using the hospice's hydrotherapy pool. Changes to the hydrotherapy processes and team have enabled appropriate access to the facility and increased use by patients.

\section{P-171 ANALYSING THE USE AND COST-EFFECTIVENESS OF DIAGNOSTIC ULTRASOUND IN A HOSPICE AND COMMUNITY SETTING}

${ }^{1,2}$ Nikki Reed, ${ }^{1}$ Kath Newton, 'Zameer Begum. 'Marie Curie West Midlands Hospice, Solihull, UK; ' University Hospitals Birmingham NHS Foundation Trust, Birmingham, UK

\subsection{6/bmispcare-2019-HUKNC.193}

Background Ultrasound is a diagnostic tool that can support clinical examination. Its use across the hospice and community palliative care setting can lead to significant cost savings with the avoidance of a hospital admission and/or the cost of hospital based interventional radiology service. Over the course of four years, 150 patients were clinically assessed with ultrasound by members of the hospice medical team (who had all received training in focussed abdominal ultrasound) and comprehensive details were recorded of their assessments.

Aim

- To ascertain if a hospice-based paracentesis service helped to reduce hospital admissions and/or interventional radiology input; 